\title{
Mass fractal characteristics of wet sonogels as determined by small-angle x-ray scattering and differential scanning calorimetry
}

\author{
D. R. Vollet, D. A. Donatti, A. Ibañez Ruiz, and F. R. Gatto \\ Departamento de Física, Unesp-Univerisdade Estadual Paulista, IGCE, P.O. Box 178 CEP 13500-970 Rio Claro, SP, Brazil
}

(Received 10 March 2006; revised manuscript received 30 May 2006; published 31 July 2006)

\begin{abstract}
Low density silica sonogels were prepared from acid sonohydrolysis of tetraethoxysilane. Wet gels were studied by small-angle x-ray scattering (SAXS) and differential scanning calorimetry (DSC). The DSC tests were carried out under a heating rate of $2{ }^{\circ} \mathrm{C} / \mathrm{min}$ from $-120^{\circ} \mathrm{C}$ up to $30^{\circ} \mathrm{C}$. Aerogels were obtained by $\mathrm{CO}_{2}$ supercritical extraction and characterized by nitrogen adsorption and SAXS. The DSC thermogram displays two distinct endothermic peaks. The first, a broad peak extending from about $-80{ }^{\circ} \mathrm{C}$ up to practically $0{ }^{\circ} \mathrm{C}$, was associated to the melting of ice nanocrystals with a crystal size distribution with "pore" diameter ranging from 1 or $2 \mathrm{~nm}$ up to about $60 \mathrm{~nm}$, as estimated from Thomson's equation. The second, a sharp peak with onset temperature close to $0{ }^{\circ} \mathrm{C}$, was attributed to the melting of macroscopic crystals. The DSC incremental "nanopore" volume distribution is in reasonable agreement with the incremental pore volume distribution of the aerogel as determined from nitrogen adsorption. No macroporosity was detected by nitrogen adsorption, probably because the adsorption method applies stress on the sample during measurement, leading to a underestimation of pore volume, or because often positive curvature of the solid surface is in aerogels, making the nitrogen condensation more difficult. According to the SAXS results, the solid network of the wet gels behaves as a mass fractal structure with mass fractal dimension $D=2.20 \pm 0.01$ in a characteristic length scale below $\xi=7.9 \pm 0.1 \mathrm{~nm}$. The mass fractal characteristics of the wet gels have also been probed from DSC data by means of an earlier applied modeling for generation of a mass fractal from the incremental "pore" volume distribution curves. The results are shown to be in interesting agreement with the results from SAXS.
\end{abstract}

DOI: 10.1103/PhysRevB.74.024208

PACS number(s): 61.10.Eq, 81.70.Pg, 68.43.- h, 61.43.Hv

\section{INTRODUCTION}

A large variety of glass and glass ceramics has been obtained by sol-gel process from the hydrolysis of tetraethoxysilane (TEOS). ${ }^{1}$ Sonochemistry ${ }^{2}$ is an effective method to promote hydrolysis of TEOS without the necessity of using mutual homogenizing solvents for water and TEOS.

Wet gels frequently exhibit structures with mass fractal features consisting of a continuous solid network imbibed in an up to almost $95 \%$-volume liquid phase. The development of mass fractal structures in wet sonogels has been well established by means of a kinetic study at different temperatures of the aggregation process of very small primary silica particles produced in the TEOS sonohydrolysis. ${ }^{3}$ Supercritical drying is one of the usual methods in dried gels production (aerogels). The structure of aerogels are often similar to that of the original wet gels, but by the contrast variation resulting from the liquid phase removal and the developing of an apparent secondary mass and/or surface fractal structure at a high resolution level. ${ }^{4}$

In general, different structures for gels have been reported depending on the starting materials, the initial conditions of preparation as $p \mathrm{H}$, alcoxide-water molar ratio, type of catalyst, temperature, and method employed for hydrolysis (ultrasound or conventional), and also on the conditions of gelation, aging, and drying of the gels. The characteristics of the structure of the gels are important for a variety of applications. Silica gels have been considered as appropriated matrices for the preparation of complex-center doped materials for a variety of metallic ions $\mathrm{s}^{5,6}$ and for encapsulation of a variety of organic ${ }^{7-9}$ and inorganic compounds, ${ }^{10,11}$ with interesting optical and/or electronic properties. The mesopo- rous structure has been considered as an important transport medium for a variety of applications such as controlledrelease carrier implantable materials for low weight drugs in biological systems ${ }^{12,13}$ and as substitute materials for membrane processes in fuel cells. ${ }^{14}$

Most of the structural properties of the gels are even defined at the early gelation and aging period in which the gels are yet in wet conditions. So the determination of the structural parameters of the wet gels could be important for control purposes of the final product. Small-angle x-ray scattering (SAXS) has successfully been applied to study the structure of wet gels (and also of aerogels) due to the nondestructive character of the technique. Nuclear magnetic resonance (NMR), ${ }^{15}$ small-angle neutron scattering (SANS), ${ }^{16}$ and differential scanning calorimetry (DSC) ${ }^{17}$ are also appropriated techniques to study the structure of wet gels. Nitrogen adsorption, for instance, is a very powerful technique in studying the structure of aerogels but it fails in studying wet gels, since the sample should be completely degassed for the adsorption experiment. Furthermore, different techniques employed in the gel structural characterization may lead to structural parameters of discussible similarity and, not always, a clear correlation between them is shown to be unequivocally established.

In this work, the nanostructural characteristics of wet gels, obtained from sonohydrolysis of TEOS, were studied by means of small-angle x-ray scattering (SAXS) and differential scanning calorimetry (DSC). The results are combined with the structure of a corresponding aerogel as determined by nitrogen adsorption and SAXS. Interesting mass fractal properties associated to the nanostructure of the wet sonogels are presented in agreement with the determination by either 
of both the techniques SAXS and DSC. In addition, DSC evinces a macroporosity apparently hidden under the observation by nitrogen adsorption.

\section{EXPERIMENT}

The samples were prepared from the sonohydrolysis of mixtures of $25 \mathrm{ml}$ of tetraethoxysilane, $8 \mathrm{ml}$ of distilled and deionized water and $5 \mathrm{ml}$ of $0.1 \mathrm{~N} \mathrm{HCl}$ as a catalyst. The resulting $p \mathrm{H}$ of the mixture was about 1.5-2.0. The hydrolysis was promoted during 10 min under a constant power $\left(\sim 0.7 \mathrm{~W} / \mathrm{cm}^{3}\right)$ of ultrasonic radiation. The sol was diluted in $28.8 \mathrm{ml}$ of water, in order to favor the obtaining of monolithic aerogels after supercritical drying, and sonication was continued for $2 \mathrm{~min}$ for complete homogenization. The $p \mathrm{H}$ of the resulting sol was adjusted to 4.5 by addition of $\mathrm{NH}_{4}(\mathrm{OH})$ to accelerate the gelation process. The final water/ TEOS molar ratio $r_{w}$ was equal to 22.5. The resulting sol was cast in sealed containers and kept under saturated conditions for 50 days at $40{ }^{\circ} \mathrm{C}$ for gelation and aging.

The aged wet gels were analyzed by SAXS as obtained. The liquid phase of the wet gels, a mixture of ethanol and water, was exchanged by pure water for the DSC tests since a pure solvent is necessary for the employed DSC method. Aerogels were obtained by a two step process: first, the original liquid phase of the wet sonogels was completely exchanged by pure ethanol; second, ethanol was exchanged by liquid $\mathrm{CO}_{2}$ in an autoclave followed by supercritical $\mathrm{CO}_{2}$ extraction. Samples of aerogels were studied by nitrogen adsorption and SAXS. The samples were degassed at $200{ }^{\circ} \mathrm{C}$ for $24 \mathrm{~h}$ before they were used for obtaining nitrogen adsorption isotherms.

The SAXS experiments were carried out using synchrotron radiation with a wavelength $\lambda=0.1608 \mathrm{~nm}$. The beam was monochromatized by a silicon monochromator and collimated by a set of slits defining a pin-hole geometry. ${ }^{18} \mathrm{~A}$ one-dimensional position sensitive x-ray detector was used to record the SAXS intensity, $I(q)$, as a function of the modulus of the scattering vector $q=(4 \pi / \lambda) \sin (\theta / 2)$, where $\theta$ is the scattering angle. The experimental setup allowed to get SAXS data from $q_{\min }=0.095 \mathrm{~nm}^{-1}$ to $q_{\max }=3.1 \mathrm{~nm}^{-1}$ with a resolution of $\Delta q=4.98 \times 10^{-3} \mathrm{~nm}^{-1}$. The data were corrected by the parasitic scattering and the sample attenuation and normalized by the intensity of the incident beam. No correction by the solvent scattering has been considered for the wet gels since the liquid phase can be considered as homogeneous compared to the high intensity associated to the electronic density difference between the silica particles and the liquid phase.

The DSC thermogram was obtained with a heating rate of $2{ }^{\circ} \mathrm{C} / \mathrm{min}$ in the temperature range from $-120^{\circ} \mathrm{C}$ up to $30^{\circ} \mathrm{C}$ using a commercial equipment (DSC 200 Phox Netzsch).

Nitrogen adsorption isotherms were obtained at liquid nitrogen temperature using a commercial pore and surface analyzer apparatus (ASAP 2010 Micromeritics). The data were analyzed for BET specific surface $\left(S_{\mathrm{BET}}\right)$, specific total pore volume $\left(V_{p}\right)$ through the total adsorbed volume of nitrogen at a single point close to the saturation pressure, pore mean size
TABLE I. Measured and estimated properties of the wet gel.

\begin{tabular}{ccccc}
\hline \hline$\rho\left(\mathrm{g} / \mathrm{cm}^{3}\right)$ & $\rho_{L}\left(\mathrm{~g} / \mathrm{cm}^{3}\right)$ & $\phi_{L}$ & $D$ & $\xi(\mathrm{nm})$ \\
\hline $1.04 \pm 0.01$ & 0.919 & 0.905 & $2.20 \pm 0.01$ & $7.9 \pm 0.1$ \\
\hline \hline
\end{tabular}

$\left(l_{\mathrm{BET}}=4 V_{P} / S_{\mathrm{BET}}\right)$, and incremental pore volume distribution, as determined through the classical Kelvin equation ${ }^{19}$ and the Harkins and Jura model ${ }^{19}$ for cylindrical pores.

\section{RESULTS}

Table I shows the bulk density $\rho$ of the wet gel as determined from volume and mass measurements after the aging at $40{ }^{\circ} \mathrm{C}$ for 50 days under completely sealed conditions. Assuming $100 \%$ hydrolysis and $100 \%$ polycondensation reactions after the long time of aging at $40{ }^{\circ} \mathrm{C}$ under completely sealed conditions, we expect that the liquid phase of the gels be a mixture of ethanol and water, in a proportion equal to $4:\left(r_{w}-2\right)$, respectively. Assuming an ideal mixture, the density of the liquid phase $\rho_{L}$ can be estimated for the wet gels (Table I) and, then, the volume fraction of the liquid phase $\phi_{L}$ by the equation $\phi_{L}=\left(\rho_{S}-\rho\right) /\left(\rho_{S}-\rho_{L}\right)$, where $\rho_{S}$ is the density of the solid particles (silica) of the gel, assumed as $2.2 \mathrm{~g} / \mathrm{cm}^{3}$ as frequently quoted for fused silica. Table I shows that the wet gel exhibits an equivalent "pore" volume fraction of $\phi_{L} \sim 90 \%$.

Figure 1 shows the SAXS intensity $I(q)$ as a function of the modulus of scattering vector $q$ of a sample of the wet gel compared to another of the aerogel after the supercritical $\mathrm{CO}_{2}$ extraction. Since the volume fraction of liquid phase was found to be about $90 \%$ in the wet gels, we are more properly concerned with the scattering from the solid particles of the system instead of the pores. The plot of the SAXS intensity in Fig. 1 shows characteristics of the scattering from a mass fractal structure. The mass fractal characteristics can be recognized by typical power-law decrease on $q$ of the SAXS intensity expressed by a scaling law as $I(q) \sim q^{-D}$ in a $q$ range given by $\xi \gg q^{-1} \gg a$, where $D$ is the mass fractal dimension, a physically acceptable value between $1<D<3, \xi$ is the characteristic length of the fractal structure and $a$ the characteristic length of the primary particles composing the structure. ${ }^{20}$ The SAXS intensity departs from the power-law regime at low $q$ due to the finite correlation length $\xi$ of the mass fractal structure. An approach accounting for this effect can be cast as ${ }^{21,22}$

$$
I(q)=A \sin [(D-1) \arctan (q \xi)] /\left(1+q^{2} \xi^{2}\right)^{(D-1) / 2}(D-1) q \xi,
$$

where $A$ is a constant for a given $D$ and $\xi$.

Figure 1 shows that Eq. (1) fits very well with the SAXS intensity curve of the wet gel and reasonably well with that of the aerogel. The fitting process yields $D=2.20$ and $\xi=7.9 \mathrm{~nm}$ for the wet gel (Table I) and $D=2.52$ and $\xi=5.6 \mathrm{~nm}$ for the aerogel (Table II). The mass fractal domain of the wet gel apparently extends up to above the experimental $q_{\max }$, which corresponds to a characteristic length $a=1 / q_{\max }=0.32 \mathrm{~nm}$ for the primary particle composing the 


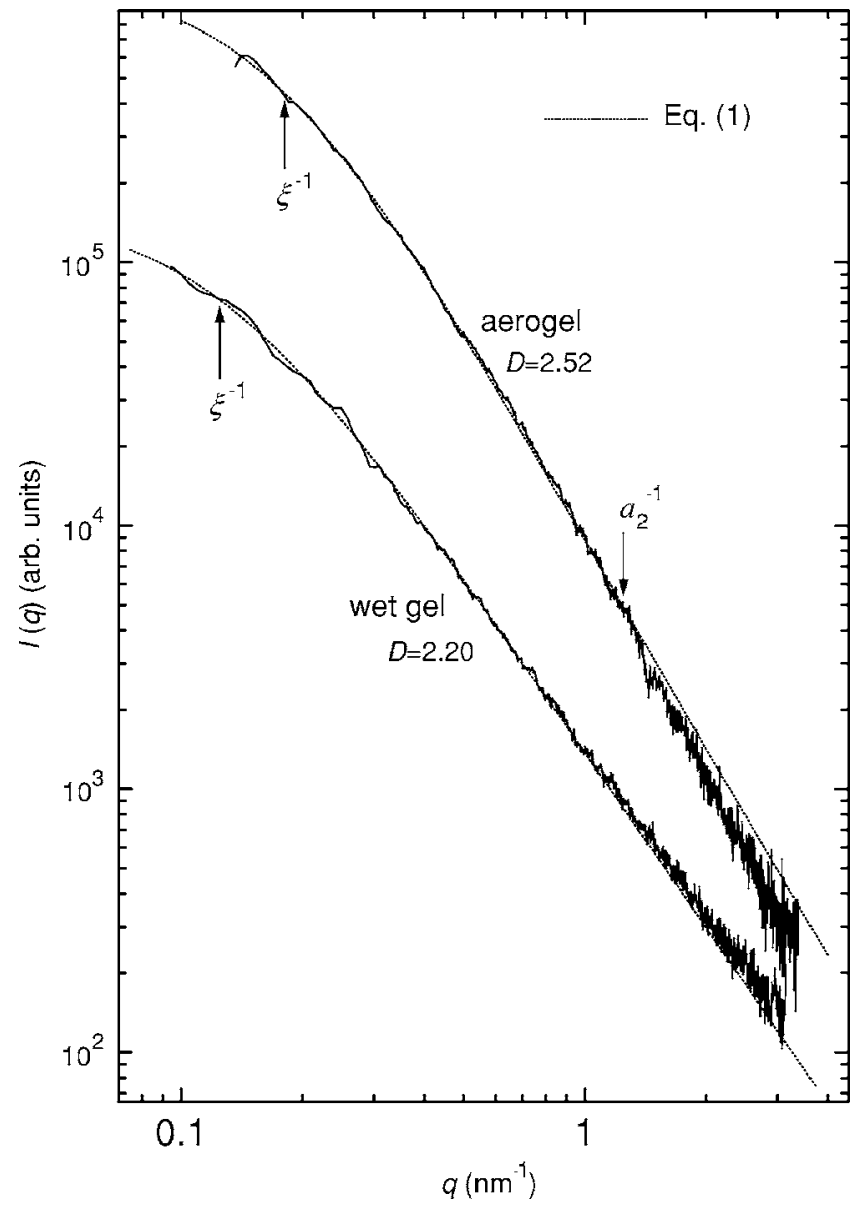

FIG. 1. SAXS intensity as a function of the modulus of scattering vector $q$ for a sample of wet gel and another of aerogel. The dotted lines represent the fitting of the mass fractal approach [Eq. (1)] to the experimental data.

fractal structure, at least. The fractality range of the wet gel should be then at least $(\xi / a) \sim 25$. We have found elsewhere ${ }^{4}$ that the fractal domain in the wet gels can extend down to $1 / q=0.22 \mathrm{~nm}$, which correspond to a Bragg distance of $\sim 1.3 \mathrm{~nm}$, a very small value to be probed by usual SAXS. The aggregation of such small size primary particles to form mass fractal structures with the typical characteristic length $\xi=7.9 \mathrm{~nm}$ (a Bragg distance of $\sim 49 \mathrm{~nm}$ ), as found in the present work, has been well established by a kinetic study of the aggregation process carried out at different temperatures. ${ }^{3}$ Since $\xi$ has been determined by the approach at the side of low angle as the crossover to a homogenous structure nonresolved by SAXS, we do not expect additional structural information below the experimental $q_{\mathrm{min}}$.

The fractality range of the mass fractal structure of the aerogel is lightly shortened with the supercritical process. $D$ was found a little greater while $\xi$ smaller than the corre-

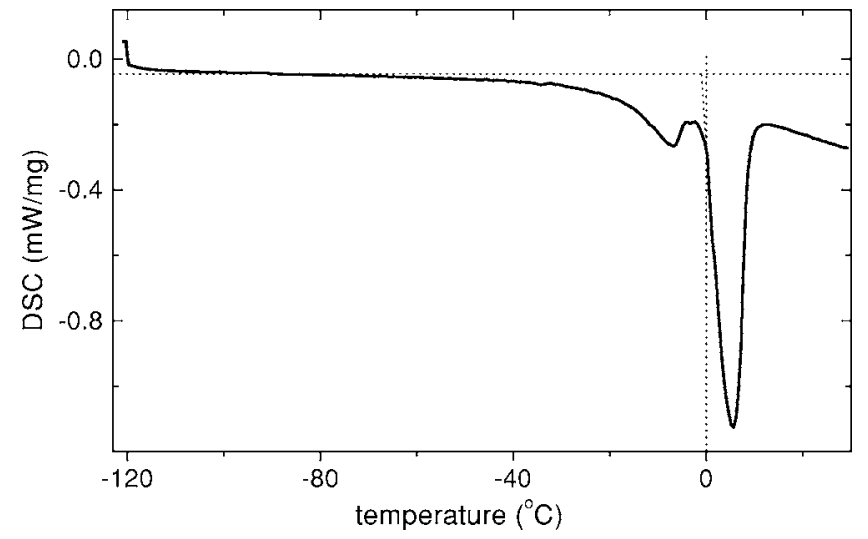

FIG. 2. DSC thermogram for a pure water exchanged wet gel carried out under a heating rate of $2{ }^{\circ} \mathrm{C} / \mathrm{min}$.

sponding values of the wet gel. Apparently at $q=a_{2}^{-1}$ $\left(a_{2} \sim 0.8 \mathrm{~nm}\right.$, corresponding to a Bragg distance of $\sim 5 \mathrm{~nm}$ ), the intensity curve of the aerogel fairly crosses over to a distinct power-law decaying $I(q) \sim q^{-\alpha}$, with the exponent $\alpha$ essentially equal to 3 . An analogous crossover to the powerlaw decaying with exponent $\alpha=3.4$ has already been observed in aerogels prepared from little more dense wet sonogels. ${ }^{4}$ It can be attributed to the formation of a new secondary particle possibly evolved from the original wet mass fractal structure, due to some structural deformation or imperfect solvent removal at a high resolution level in the supercritical process. It could also contribute to the apparently high value found for $D$ in the aerogel. As we will see, the structure of the secondary particle seems to account for the microporosity developed in the aerogels as determined by the nitrogen adsorption.

Figure 2 shows a DSC thermogram carried out under a heating rate of $2{ }^{\circ} \mathrm{C} / \mathrm{min}$ for an about $20 \mathrm{mg}$ sample of wet gel after exchange of the original liquid phase by pure water. It has been shown ${ }^{17}$ that, by using a heating rate of 2 $2{ }^{\circ} \mathrm{C} / \mathrm{min}$ or slower, the thermogram approaches to that of the equilibrium conditions for fusion of the nanocrystals of the ice in the gels, yielding true information on the "porous" structure of the wet gels.

Two endothermic melting peaks are apparent in Fig. 2. The first melting peak extending from about $-80{ }^{\circ} \mathrm{C}$ up to practically $0{ }^{\circ} \mathrm{C}$ was associated to the melting of nanocrystals of ice with a crystal size distribution in the nanometer scale. The onset temperature $-80^{\circ} \mathrm{C}$ of the melting peak of the nanocrystals was estimated from numerical determination of the apparent inflection point in the base line of the DSC thermogram. The second, a sharp peak with onset temperature close to $0{ }^{\circ} \mathrm{C}$, was associated to the melting of macroscopic ice crystals.

According to Thomson's equation, the melting depression temperature $\Delta T_{m}=T_{m}-T_{m}^{0}$ of an ice crystal with radius $r$ can be evaluated from ${ }^{17}$

TABLE II. Structural properties of the aerogel.

\begin{tabular}{cccccccc}
\hline \hline$D$ & $\xi(\mathrm{nm})$ & $S_{\mathrm{BET}}\left(\mathrm{m}^{2} / \mathrm{g}\right)$ & $V_{p}\left(\mathrm{~cm}^{3} / \mathrm{g}\right)$ & $l_{\mathrm{BET}}(\mathrm{nm})$ & $\rho_{\text {aero }}\left(\mathrm{g} / \mathrm{cm}^{3}\right)$ & $\rho_{\mathrm{N} 2}\left(\mathrm{~g} / \mathrm{cm}^{3}\right)$ & $\phi_{\mathrm{N} 2}$ \\
\hline $2.52 \pm 0.02$ & $5.6 \pm 0.1$ & $848 \pm 15$ & $1.69 \pm 0.05$ & $8.0 \pm 0.1$ & $0.33 \pm 0.02$ & $0.47 \pm 0.02$ & $0.78 \pm 0.01$ \\
\hline \hline
\end{tabular}




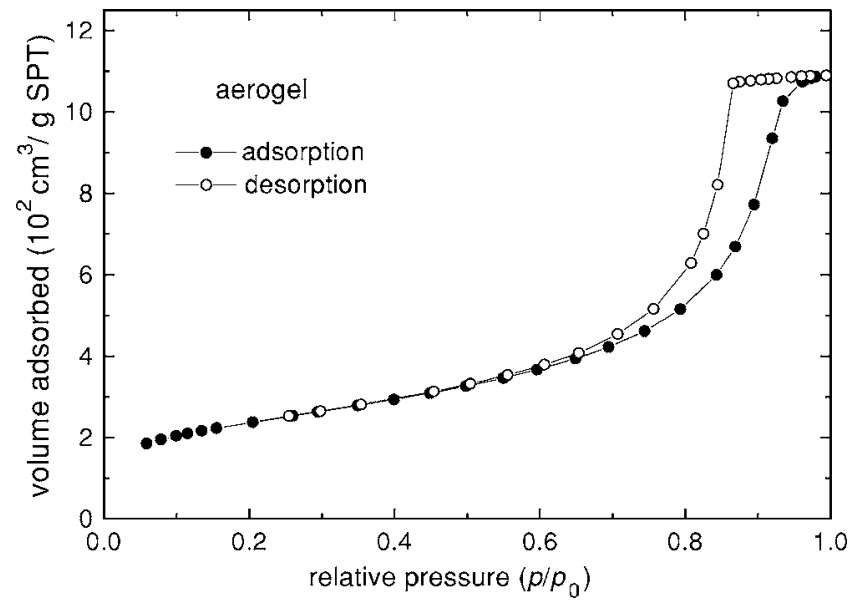

FIG. 3. Nitrogen adsorption isotherm carried out at liquid nitrogen temperature for a sample of aerogel.

$$
T_{m}=T_{m}^{0}(1-2 \gamma V / \Delta H r)
$$

where $T_{m}^{0}$ is the melting temperature of an ice crystal of infinite dimension, $\gamma$ the average interfacial tension of the crystal, $\Delta H$ the specific melting heat, and $V$ the solid specific volume. The maximum of the DSC melting peak of the nanocrystals in Fig. 2 occurs at $T_{m}=-6.8^{\circ} \mathrm{C}$, which gives a pore diameter of $2 r=20 \mathrm{~nm}$, assuming for water $^{17} T_{m}^{0}=273 \mathrm{~K}, \gamma=40 \times 10^{-3} \mathrm{~N} / m, \Delta H=334 \mathrm{~J} / \mathrm{g}$, and $V=1.02 \mathrm{~cm}^{3} / \mathrm{g}$.

Figure 3 shows the nitrogen adsorption isotherm carried out at liquid nitrogen temperature for a sample of aerogel after the supercritical extraction of $\mathrm{CO}_{2}$. The isotherm is more properly classified as type IV according to IUPAC classification. ${ }^{23}$ Table II shows the values for the specific surface $S_{\mathrm{BET}}$, the specific total pore volume $V_{p}$ and the pore mean size $l_{\mathrm{BET}}$ for the aerogel as determined from the adsorption isotherm. Table II also shows the aerogel bulk density $\rho_{\text {aero }}$ as determined from volume and mass measurements before degassing at $200{ }^{\circ} \mathrm{C}$ for the adsorption test. The nitrogen bulk density of the aerogel $\rho_{\mathrm{N} 2}$ (Table II) was estimated from $V_{p}$ as $\left(1 / \rho_{\mathrm{N} 2}\right)=\left(1 / \rho_{S}\right)+V_{p}$ assuming $\rho_{S}=2.2 \mathrm{~g} / \mathrm{cm}^{3}$ for the density of the silica particles of the aerogel. The nitrogen pore volume fraction $\phi_{\mathrm{N} 2}$ of the aerogels (Table II) was evaluated as $\phi_{\mathrm{N} 2}=\left(\rho_{S}-\rho_{\mathrm{N} 2}\right) / \rho_{S}$.

Figure 4 shows the specific incremental pore volume distribution for the aerogel as determined from the nitrogen adsorption isotherm. The maximum in the incremental pore volume distribution curve of the aerogel was found at the pore width $2 r=20 \mathrm{~nm}$, in excellent agreement with the maximum in the DSC curve. No evidence of the presence of macropores is apparent from the nitrogen adsorption data. Then, we associated the incremental pore volume distribution curve of the mesopores as determined by nitrogen adsorption to the DSC melting peak of the nanocrystals of ice. As, under the conditions close to the equilibrium, the instantaneous energy flux in the DSC test is proportional to the incremental volume of the crystals melting at the temperature $T_{m}$, then the incremental nanocrystal volume distribution can be obtained from Thomson's equation [Eq. (2)] simply as

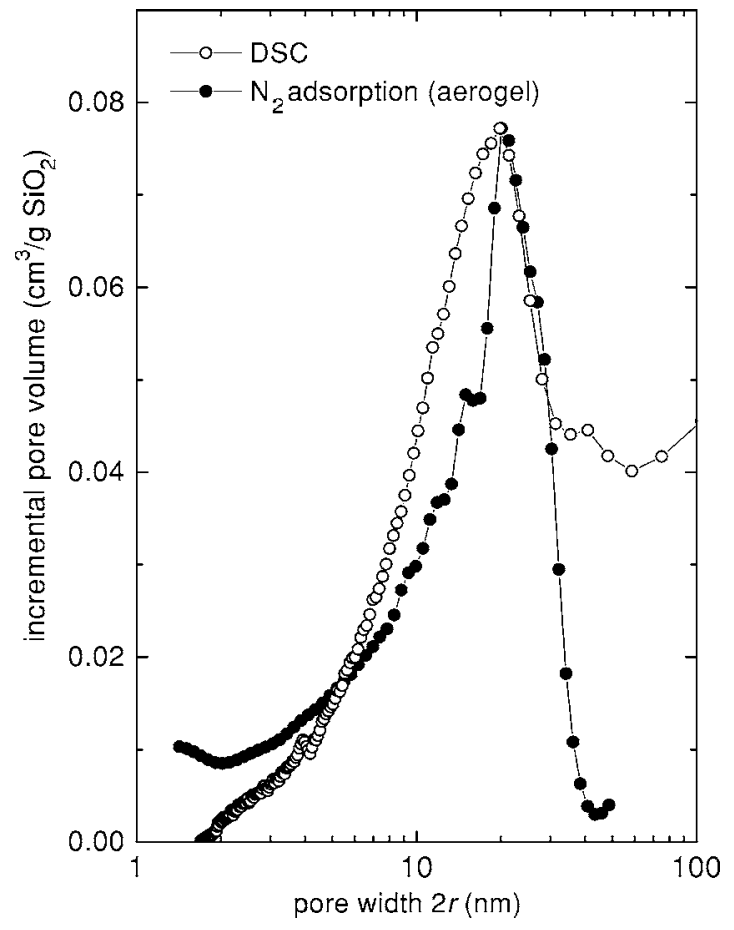

FIG. 4. Normalized incremental "pore" volume distribution of the wet gel as determined from DSC through Thomson's equation compared to the incremental pore volume distribution of the aerogel as determined from nitrogen adsorption.

a value proportional to the instantaneous DSC signal, under the conditions of constant heating rate and melting enthalpy. Due to the difficulty for the exact base line definition in the DSC curve for normalization purposes, the curve of the incremental nanocrystal volume distribution by DSC was normalized by the maximum values found at the "pore" width $20 \mathrm{~nm}$, which was matched for both DSC and nitrogen adsorption. Figure 4 shows the normalized incremental nanocrystal volume distribution of the wet gel compared to the incremental pore volume distribution of the aerogel.

\section{DISCUSSION}

The incremental nanocrystal volume distribution of the wet gel and the incremental pore volume distribution of the aerogel are in reasonable agreement, mainly with respect to the value $20 \mathrm{~nm}$ of the "pore" width associated to the maximum in both the distributions. However, it is not apparent from the nitrogen adsorption study the existence of any macroporosity as suggested by the melting peak with onset temperature close to $0{ }^{\circ} \mathrm{C}$ detected by DSC. The "pore" volume fraction of the wet gel was estimated to be $\phi_{L} \sim 0.90$ (Table I) and the nitrogen pore volume fraction of the aerogel $\phi_{\mathrm{N} 2} \sim 0.78$ (Table II). Such a difference seems not to be enough to account for the great DSC melting peak associated to the macropores. An independent estimate of the pore volume fraction of the aerogel using the measured value $\rho_{\text {aero }}=0.33 \mathrm{~g} / \mathrm{cm}^{3}$ in Table II through $\left(\rho_{S}-\rho_{\text {aero }}\right) / \rho_{S}$ yields $\phi=0.85$, which is a value more close to the value 0.90 of the wet gel. Even the measured value $\rho_{\text {aero }}=0.33 \mathrm{~g} / \mathrm{cm}^{3}$ in 
Table II could be superestimated due to the water reabsorption by the aerogel during the bulk density measurement, so the pore volume fraction in the aerogel could be even greater. As a matter of fact, if we double the specific pore volume $V_{p}$ in Table II $\left(1.69 \mathrm{~cm}^{3} / \mathrm{g}\right)$ assuming hypothetically the value $3.38 \mathrm{~cm}^{3} / \mathrm{g}$ for the specific pore volume in the aerogel, it would yield a pore volume fraction $\phi=0.88$, which would yet be a minor value than but more close to that 0.90 of the wet gel. The overall results of DSC and nitrogen adsorption would be in agreement if we realize that nitrogen adsorption induces an underestimation of the macroporosity during measurement.

Scherer and co-workers ${ }^{24,25}$ have shown that, in the case of low density aerogels, the adsorption method applies stress on the sample during measurement leading to an underestimation of pore volume. In such studies, direct experimental observation and results from modeling have shown that the volume of low density silica aerogels [less than about $0.35 \mathrm{~g} / \mathrm{cm}^{3}$ as a reference value (compare to $\rho_{\text {aero }}$ $=0.33 \mathrm{~g} / \mathrm{cm}^{3}$ in Table II)] can shrink up to about $50 \%$ as liquid nitrogen condenses in the pores. In addition to the hypothesis of underestimation of the macroporosity, they have also shown ${ }^{26,27}$ that nitrogen should not necessarily fill all the macropores in nitrogen adsorption experiments in the case of aerogels, since most of the solid surface of the aerogel has positive curvature, making the nitrogen condensation more difficult. Further, the macroporosity could be underestimated in the nitrogen adsorption.

We compare the mass fractal characteristics of the wet gels as determined by SAXS with the mass fractal structure resulting from the network silica imbibed in the incremental "pore" volume distribution as determined from DSC. For this, we have applied an earlier employed $\operatorname{method}^{28}$ in an analogy to a method used to generate nonrandom fractals, starting from an initial configuration by a sequence of approximants. Experimentally, we start from a homogeneous solid with density $\Delta \rho_{S}=\rho_{S}-\rho_{\text {ice, }}$, where $\rho_{S}$ is the silica density and $\rho_{\text {ice }}$ the density of the ice, assumed as $0.971 \mathrm{~g} / \mathrm{cm}^{3}$, and follow incorporating to the structure, step by step, each incremental "pore" volume per mass unit of such a solid $\left[\delta V_{P}\left(r_{i}\right)\right]$, associated to the "pore" class with radius $r_{i}$, starting from $r_{i}=0$ up to $r_{i}=r$, and probing the evolution of the bulk density $\Delta \rho(r)$ of such a solid, with respect to the ice density. The process can be cast as

$$
\frac{1}{\Delta \rho(r)}=\frac{1}{\Delta \rho_{S}}+\sum_{r_{i}=0}^{r_{i}=r} \delta V_{p}\left(r_{i}\right) .
$$

For a mass fractal structure of an aerogel (empty pores) defined in a length scale range $a \leqslant r \leqslant \xi$, the aerogel bulk density $\rho_{\xi}$ can be cast ${ }^{29}$ as

$$
\rho_{\xi}=\rho_{S}(\xi / a)^{D-3} .
$$

Therefore, the bulk density of our "wet" structure $\Delta \rho(r)$ should scale in the range $a \leqslant r \leqslant \xi$ as

$$
\Delta \rho(r)=\Delta \rho_{S}(r / a)^{D-3} \text {. }
$$

According to Eq. (5), the plot on a log-log scale of $\Delta \rho(r)$ as function of the structure length scale $r$ should be a straight

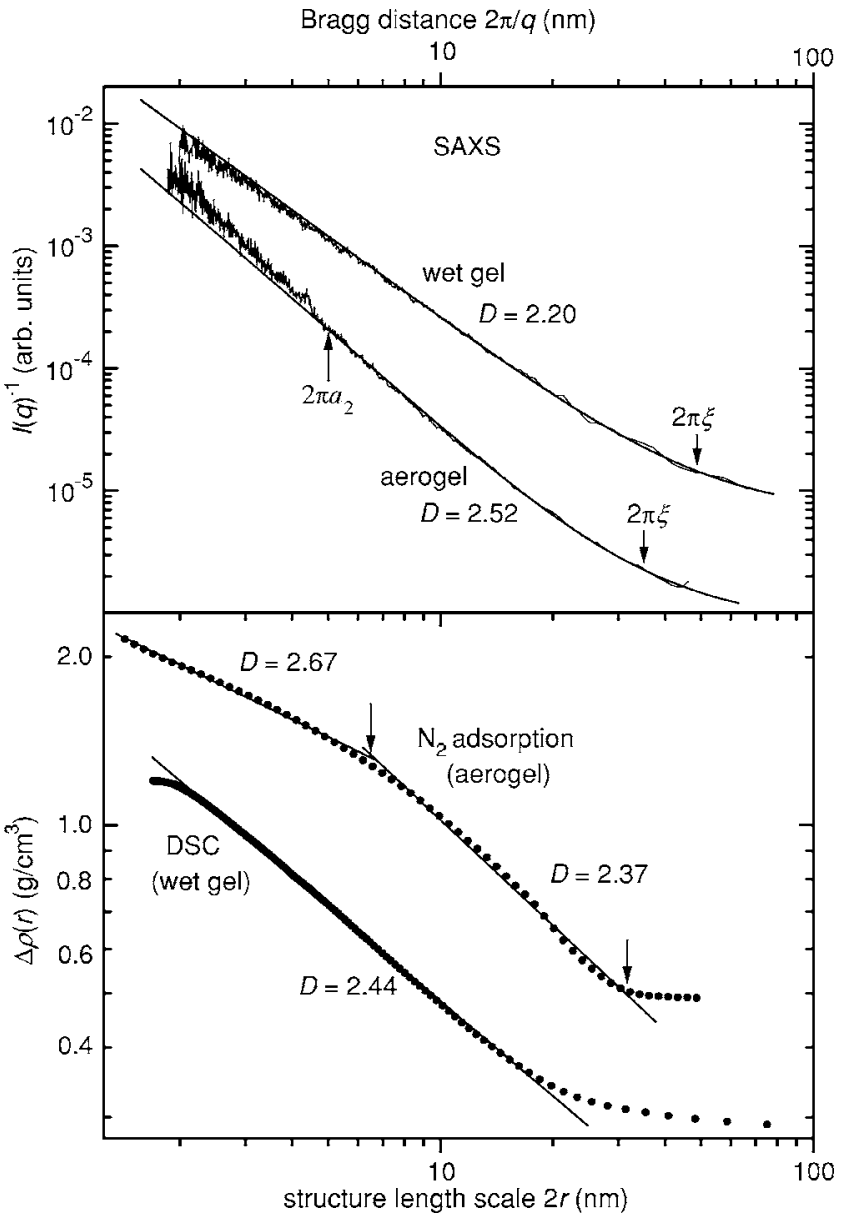

FIG. 5. Mass fractal characteristics of the wet gel (evaluated from the DSC "pore" volume distribution) and of the aerogel (evaluated from the nitrogen adsorption pore volume distribution) compared to those as obtained from SAXS. The SAXS data were plotted as $I(q)^{-1}$ versus $2 \pi / q$, the Bragg distance associated to the reciprocal of the modulus of the scattering vector $q$, in order to direct comparison with the real structure length scale as probed by the pore width $2 r$.

line with slope $D-3$. Figure 5 [DSC (wet gel)] shows the plot of $\Delta \rho(r)$ on a log-log scale with the structure length scale as probed by the "pore" width $2 r$, as evaluated through Eq. (3) from the DSC incremental "pore" volume distribution shown in Fig. 4. Figure 5 shows Eq. (5) fitting reasonably well the experimental $\Delta \rho(r)$ in a length scale of "pore" width below about $20 \mathrm{~nm}$. The slope $D-3$ of the corresponding linear fitting yields $D=2.44$ for the mass fractal dimension of the pure water exchanged wet gel. This value is a little greater than that 2.20 as obtained from SAXS for the original wet gel. The difference could be due to the exchange of the original liquid phase of the gel, a mixture of ethanol and water, by the pure water, which presents greater surface tension, so proportioning additional stress on the sample possibly leading to some additional sample contraction. The SAXS curve and the fitting of the mass fractal approach given by Eq. (1) was plotted in Fig. 5 as $I(q)^{-1}$ versus Bragg distance $2 \pi / q$ for a direct comparison. The fractality range is in reasonable agreement if we assign the "pore" width 
probed by DSC to the Bragg distance associated to the characteristic length $1 / q$ probed by SAXS.

The mass fractal of the aerogel as determined by SAXS was also compared to that as determined from nitrogen adsorption from the mesopore size distribution. The function $\rho_{r}$ associated to the aerogel bulk density was obtained from Eq. (3) (evidently changing $\rho_{\text {ice }}$ by $\rho_{\text {pore }}=0$ ), using the mesopore size distribution of aerogel in Fig. 4. The function $\Delta \rho(r)=\rho_{r}$ for the aerogel is shown in Fig. $5\left[\mathrm{~N}_{2}\right.$ adsorption (aerogel)] together with $\Delta \rho(r)$ for the wet gel in comparison to the SAXS results. Equation (5) fits fairly $\rho_{r}$ in a mesopore region, approximately between $2 r \sim 32 \mathrm{~nm}$ and $2 r \sim 6.5 \mathrm{~nm}$, with $D=2.37$, and in a micropore region, below about $2 r \sim 6.5 \mathrm{~nm}$, with $D=2.67$. The value $2 r \sim 32 \mathrm{~nm}$ is in notable agreement with the Bragg distance $2 \pi \xi \sim 35 \mathrm{~nm}$ as probed by SAXS while the apparent crossover at $2 r \sim 6.5 \mathrm{~nm}$ was found interestingly close to the Bragg distance $2 \pi a_{2} \sim 5 \mathrm{~nm}$, accounting for the secondary particle evolved with the supercritical process as suggested by SAXS. The structure of the secondary particle can then be interpreted as a more compact mass fractal $(D=2.67)$ with respect to that associated to the mesopore region $(D=2.37)$, which has been evolved in the aerogel with the supercritical process and degassing the sample at $200{ }^{\circ} \mathrm{C}$. Thus SAXS and nitrogen adsorption techniques seem to describe the same structure for the aerogel but from completely independent physical principles.

The macroporosity was detected by DSC. Certainly the macroporosity is not detected by SAXS and so it appears as nitrogen adsorption. Now, we are preparing a set of samples of sonogels with different volume fraction of macroporosity to probe its influence on the overall structure of the sonogels applying the present methodology. It could be also interesting to study the structure behavior on the cooling of the samples.

Finally, we wonder if the mass fractal dimension obtained in this work by SAXS and DSC for wet gels (2.20 and 2.44) could be compared to the exponent $D^{*}$ that would be obtained under a hypothetical condition that the value $V(r)$ $=\sum_{r_{i}=0}^{r_{i}=r} \delta V_{P}\left(r_{i}\right)$ [the sum in Eq. (3)] would follow a power law on $r$ as $V(r) \sim r^{D^{*}}$. No linear relation was observed by plotting the sum $V(r)$ against $r$ on a $\log -\log$ scale. However, Neffati and Rault ${ }^{17}$ have shown that, under the condition of a pore size distribution $P(r)=d V(r) / d r$ following a power law $d V(r) / d r \sim r^{\left(D^{*}-1\right)}$ [a differential form of the power law $\left.V(r) \sim r^{D^{*}}\right]$, the heat flow $(J)$ of the DSC signal is related to the modulus of the melting depression temperature $\Delta T_{m}$ by a scaling law as $J \sim \Delta T_{m}^{-\left(D^{*}+1\right)}$. Figure 6 shows the plot of the DSC signal against the modulus of $\Delta T_{m}$ on a log-log scale. A fairly linear relation is apparent in the $\Delta T_{m}$ domain between approximately $10^{\circ} \mathrm{C}$ and $60^{\circ} \mathrm{C}$, which corresponds to "pore" width of $\sim 13 \mathrm{~nm}$ and $\sim 2.2 \mathrm{~nm}$, respectively. This domain is in reasonable agreement with the plots in Fig. 5, however, the slope $-\left(D^{*}+1\right)$ of the linear fitting in Fig. 6 yields $D^{*}=0.63$, which is apparently not in agreement with the mass fractal dimension $D$ shown in Fig. 5. The reason for this could be due to the fact that our incremental "pore" volume distribution shown in Fig. 5 has dimension of spe-

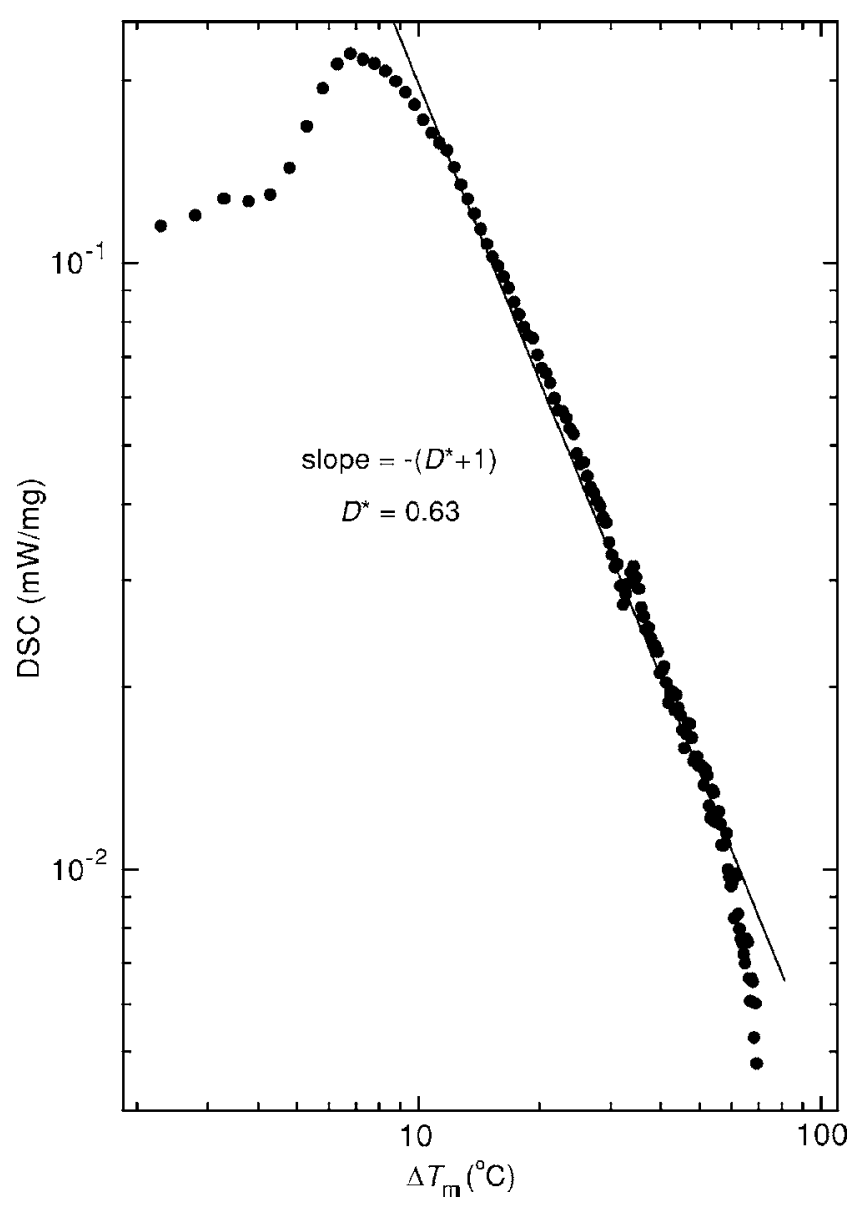

FIG. 6. Instantaneous DSC heat flow as a function of the modulus of the melting depression temperature.

cific volume $\left(\mathrm{cm}^{3} / \mathrm{g}\right)$ and does not match the pore size distribution $P(r)$ used in the fundamentals of the Neffati and Rault modeling, which has dimension of specific surface $[d V(r) / d r]$. Then, we think both the parameters have different physical meaning.

\section{CONCLUSIONS}

Silica gels prepared from sonohydrolysis of TEOS with low density of silica present a bimodal pore structure constituted by nanopores and macropores, which display two distinct melting peaks in a DSC thermogram.

The incremental "pore" volume distribution of the nanopores in wet gels can be obtained from the corresponding DSC melting peak through Thomson's equation, which establishes a relation between the melting depression temperature and the radius of the nanocrystal.

The DSC incremental "nanopore" volume distribution in the wet gel is in reasonable agreement with the incremental pore volume distribution of the corresponding aerogel after supercritical drying, as obtained from nitrogen adsorption. No macroporosity could be detected by nitrogen adsorption in this low density aerogel, probably due to the fact that the adsorption method applies stress on the sample during measurement, leading to an underestimation of pore volume, or 
due to the positive curvature of the solid surface often associated to the macropores, making the nitrogen condensation more difficult. The general characteristics of the mass fractal of the aerogel as determined by SAXS are in interesting agreement with those evaluated from the pore volume distribution by adsorption.

The structure of the wet gel behaves as a mass fractal structure with mass fractal dimension $D=2.20$ in a length scale below about $\xi=7.9 \mathrm{~nm}$, as determined by SAXS. The application of an earlier model for the generation of a mass fractal structure from the DSC incremental "pore" volume distribution yielded $D=2.44$ for the pure water exchanged wet gel, in a fractality range below about $20 \mathrm{~nm}$, which is in reasonable agreement with the SAXS result, if we assign the "pore" diameter probed by DSC to the Bragg distance associated to the characteristic length $1 / q$ probed by SAXS.

\section{ACKNOWLEDGMENTS}

Research was partially supported by LNLS - National Synchrotron Light Laboratory, FAPESP and CNPq.
${ }^{1}$ C. J. Brinker and G. W. Scherer, Sol-Gel Science: The Physics and Chemistry of Sol-Gel Processing (Academic, San Diego, 1990).

${ }^{2}$ M. Tarasevich, Am. Ceram. Soc. Bull. 63, 500 (1984).

${ }^{3}$ D. R. Vollet, D. A. Donatti, and A. Ibañez Ruiz, J. Non-Cryst. Solids 288, 81 (2001).

${ }^{4}$ D. R. Vollet, D. A. Donatti, A. Ibañez Ruiz, and W. C. de Castro, J. Non-Cryst. Solids 332, 73 (2003).

${ }^{5}$ M. Morita, S. Kajiyama, D. Rau, T. Sakurai, and M. Iwamura, J. Lumin. 102-103, 608 (2003).

${ }^{6}$ P. Sujatha Devi and D. Ganguli, J. Non-Cryst. Solids 336, 128 (2004).

${ }^{7}$ R. Litrán, M. Ramírez-del-Solar, and E. Blanco, J. Non-Cryst. Solids 318, 49 (2003).

${ }^{8}$ R. Litrán, E. Blanco, and M. Ramírez-del-Solar, J. Non-Cryst. Solids 333, 327 (2004).

${ }^{9}$ A. Parvathy Rao and A. Venkateswara Rao, Sci. Technol. Adv. Mater. 4, 121 (2003).

${ }^{10}$ W. Chen, J. Zhang, and W. Cai, Scr. Mater. 48, 1061 (2003).

${ }^{11}$ Y. Feng, R. Yao, and L. Zhang, Physica B 350, 348 (2004).

${ }^{12}$ M. Ahola, P. Kortesuo, I. Kangasnieme, J. Kiesvaara, and A. YliUrpo, Int. J. Pharm. 195, 219 (2000).

${ }^{13}$ S. Radin, G. El-Bassyouni, E. J. Vresilovic, E. Schepers, and P. Ducheyne, Biomaterials 26, 1043 (2005).

${ }^{14}$ M. T. Colomer and M. A. Anderson, J. Non-Cryst. Solids 290, 93 (2001).

${ }^{15}$ F. Devreux, J. P. Boilot, F. Chaput, and B. Sapoval, Phys. Rev.
Lett. 65, 614 (1990).

${ }^{16}$ J. C. Li, D. K. Ross, and R. K. Heenan, Phys. Rev. B 48, 6716 (1993).

${ }^{17}$ R. Neffati and J Rault, Eur. Phys. J. B 21, 205 (2001).

${ }^{18}$ G. Kellermann, F. Vicentin, E. Tamura, M. Rocha, H. Tolentino, A. Barbosa, A. Craievich, and I. Torriani, J. Appl. Crystallogr. 30, 880 (1997).

${ }^{19}$ S. J. Gregg and K. S. W. Sing, Adsorption, Surface Area and Porosity (Academic, New York, 1967).

${ }^{20}$ D. W. Schaefer and K. D. Keefer, Phys. Rev. Lett. 53, 1383 (1984).

${ }^{21}$ T. Freltoft, J. K. Kjems, and S. K. Sinha, Phys. Rev. B 33, 269 (1986).

${ }^{22}$ R. Vacher, T. Woignier, J. Pelous, and E. Courtens, Phys. Rev. B 37, 6500 (1988).

${ }^{23}$ G. Leofanti, M. Padovan, G. Tozzola, and B. Venturelli, Catal. Today 41, 207 (1990).

${ }^{24}$ G. W. Scherer, D. M. Smith, and D. Stein, J. Non-Cryst. Solids 186, 309 (1995).

${ }^{25}$ G. W. Scherer, J. Non-Cryst. Solids 225, 192 (1998).

${ }^{26}$ G. W. Scherer, J. Colloid Interface Sci. 202, 399 (1998).

${ }^{27}$ G. Reichenauer and G. W. Scherer, J. Colloid Interface Sci. 236, 385 (2001).

${ }^{28}$ D. R. Vollet, D. A. Donatti, A. Ibañez Ruiz, Phys. Rev. B 69, 064202 (2004).

${ }^{29}$ T. Woignier, J. Phalippou, R. Vacher, J. Pelous, and E. Courtens, J. Non-Cryst. Solids 121, 198 (1990). 